\section{EVALUACIÓN CRÍTICA DE GOBIERNO ELECTRÓNICO COMO HERRAMIENTA PARA EL CAMBIO DE LA ESTRUCTURA GUBERNAMENTAL}

\author{
CRITICAL EVALUATION OF ELECTRONIC GOVERNMENT AS A TOOL \\ FOR CHANGING THE GOVERNMENTAL STRUCTURE
}

Johandry N. López U.

\section{Resumen}

El avance de las tecnologías y de las comunicaciones a nivel mundial, la velocidad con la que se realizan distintos tipos de operaciones, a través de la utilización de Internet en el ámbito privado, y las ventajas que todo esto implica provocan en los ciudadanos un cambio fundamental en la forma habitual de relacionarse, es por ello, que el Estado debe adecuarse a las nuevas tendencias por tanto, surge así lo que se ha dado a conocer como Gobierno Electrónico (GE), pero ¿sabemos qué es?, ¿cómo ha sido su incidencia a nivel internacional? ¿Cuál ha sido su importancia y aportes la sociedad venezolana? Para poder dar respuestas a las siguientes interrogantes se realizó un estudio exploratorio haciendo una revisión exhaustiva de más de 40 publicaciones realizadas, instituciones de trayectoria a nivel mundial y entrevistas informales con profesionales vinculados a las TIC en Venezuela. Para finalizar los modelos teóricos existentes para identificar la madurez del país entorno a tema, así como los diez indicadores claves y los siete extendidos propuestos por el Grupo TIC de la CEA y la OSILAC y por último la medición del ranking de desarrollo de GE para establecer la relación entre las administraciones nacionales y la prestación de servicios públicos con el uso de las TIC, adoptado para la medición del desarrollo de gobierno electrónico en Venezuela.

Palabras clave: Gobierno Electrónico; TIC; Gestión Pública

\begin{abstract}
The advancement of technologies and communications worldwide, the speed with which different types of operations are carried out, through the use of the Internet in the private sphere, and the advantages that all this implies cause a change in citizens fundamental in the usual way of relating, that is why the State must adapt to the new trends, therefore, what has become known as Electronic Government (GE) arises, but do we know what it is?, how has it Was its incidence internationally? What has been its importance and contributions of Venezuelan society? To be able to answer the following questions, an exploratory study was carried out, making an exhaustive review of more than 40 publications made, trajectory institutions worldwide and informal interviews with professionals related to ICT in Venezuela. To finalize the existing theoretical models to identify the maturity of the country around the subject, as well as the ten key indicators and the seven extended ones proposed by the ICT Group of the CEA and OSILAC and finally the measurement of the GE development ranking to establish the relationship between national administrations and the provision of public services with the use of ICT, adopted for measuring the development of electronic government in Venezuela.
\end{abstract}

Key words: Electronic Government; ICT; Public Management

Artículo recibido 19 enero 2017 | Arbitrado febrero 2017 | Publicado 01 de abril 2017

\section{Johandry N. López U.}

johandry.gomes@gmail.com

Observatorio Nacional de Ciencia, Tecnología e Innovación (ONCTI), Venezuela

Doctoranda en Gestión de la Investigación y Desarrollo en la Universidad Central de Venezuela 2019, recibida como Especialista en Gerencia de Proyectos en la Universidad Católica Andrés Bello en el año 2009, realizó un Diplomado en Gestión de Gobierno Electrónico en la Universidad Dr. José Gregorio Hernández en el año 2014, y Egresada como Licenciada en Ciencias Estadísticas en la Universidad Central de Venezuela en el año 2006. Ha realizado actividades vinculadas a la Ciencia, Tecnología e Innovación a lo largo de 14 años en donde los proyectos más relevantes en su trayectoria laboral ha sido la Formulación y Aplicación de Indicadores de Ciencia y Tecnología en el Marco del ALBA-TCP, Diseño Metodológico para la Gestión de Proyectos de la UCAB enmarcados en las directrices de la LOCTI, Participación en la realización del boletín de Indicadores Venezolanos de Ciencia, Tecnología e Innovación, así como el cumplimiento con instituciones nacionales e internacionales. 


\section{INTRODUCCIÓN}

Si a nivel de Gobernanza venezolana se refiere, es imperativa la implementación de mecanismos que permitan disponer de herramientas tecnológicas para facilitar $y$ mejorar los procesos de gobernabilidad $\mathrm{y}$ administración pública que a su vez de cabida a la aceleración de oportunidades que los sistemas de Gobierno Electrónico brindan a sus habitantes, es por ello que las TIC se considera como herramienta con un importante potencial para configurar estructuras organizativas y modelos de gestión que ofrezcan una respuesta única, ágil, eficiente, de calidad y transparente a la población. De esta manera la extensión y el uso de las TIC y la creación de iniciativas y proyectos de Gobierno Electrónico, como anteriormente pudieron serlo las ideas de receptividad de la Administración, orientación al ciudadano, calidad y mejora continua en la prestación de servicios, podrían ser un motor de cambio para las organizaciones existiendo también la amenaza de que sea o se convierta en una nueva moda.

Es por ello que en Venezuela, es necesario realizar una evaluación crítica de gobierno electrónico como herramienta para el cambio de la estructura gubernamental comenzando por su concepción a nivel de gobierno y gestión pública, el conocimiento de Gobierno Electrónico a nivel internacional comenzando con países desarrollados, cuya implementación ha tenido una incidencia directa con el ciudadano común y una mejora significativa en el control $y$ tramitación exitosas sumado a bajo costo e impacto ambiental positivo también resulta interesante conocer cómo ha sido la estructura a nivel de América Latina en cuanto a la implementación y desarrollo de Gobierno Electrónico, sí como el manejo internacional de indicadores en TIC relacionados al tema que incluye el ranking de GE y el posicionamiento de Venezuela como país.

Sumado al escenario antes descrito está la dinámica venezolana que es diferente a la de otros países hermanos debido a la transición entre el capitalismo y el socialismo bolivariano del siglo XXI, en donde se preserva la igualdad, solidaridad y la complementariedad tanto a lo interno como las relaciones entre las naciones, por lo tanto es crucial la identificación de los beneficios de Gobierno Electrónico adaptados a nuestra doctrina política e ideológica, así como el modelo adoptado en la República Bolivariana de Venezuela para la medición de gobierno electrónico.

\section{A nivel internacional}

\section{¿Sabemos lo que es gobierno electrónico?}

A nivel internacional no existe una definición universal de Gobierno Electrónico, ya que en cada uno de los países en las que ha sido implementada ha sufrido transformaciones que responde a su realidad, sin embargo, haciendo una revisión a nivel internacional se ha hallado posturas interesantes, pero sólo una describe específicamente lo que se pretende de él en un país, según las Cimoli y CEPAL (2005) el Gobierno Electrónico se define en función de quién haga uso de él debido a que, desde un punto de vista tecnológico, el Gobierno Electrónico es la capacidad que ofrecen las TIC para desarrollar y ofrecer información y servicios públicos a través de medios telemáticos, habitualmente Internet. Para el Gobierno, el GE es el medio para modernizar la gestión pública a través de las TIC, en busca de mejores prácticas, mayor control yo 
transparencia, y más agilidad. En definitiva, una mejor prestación de servicios al país en su conjunto. $Y$ por último para los usuarios del GE, es la oportunidad para acceder y participar activamente en los servicios públicos, de forma más flexible y sin acudir a las dependencias del Estado. También significa más información, más puntual y a cualquier hora.

Las características más resaltantes de Gobierno Electrónico son:

- E-Gobierno es un gobierno que utiliza las tecnologías de la información y las comunicaciones (TIC) para transformar sus relaciones internas y externas.

- Al utilizar las TIC el gobierno no altera sus funciones básicas o su obligación de ser útil, legítimo, transparente $\mathrm{y}$ responsable.

- Las aplicaciones de E-Gobierno aumentan las expectativas de desempeño y resultado público por parte de la Sociedad.

\section{¿Cómo es la funcionalidad de gobierno electrónico?}

- Transparencia (Monitoreo al Gobierno...): Nivel de apego de la acción gubernamental a los principios legales y éticos, así como al compromiso de la entidad de hacer partícipe a la ciudadanía de los planes implementados y de los resultados obtenidos.

- Eficacia ¿lo estamos logrando?: Niveles de cumplimiento de la entidad respecto de sus objetivos institucionales y metas previstas.

- Eficiencia ¿lo estamos logrando de la mejor manera?: Balance entre los costos de cada acción pública y los beneficios que serán generados.
- Acceso (disminuir la brecha digital): Nivel de disponibilidad y facilidad de uso de los distintos servicios ofrecidos por el sistema público acortando distancias y borrando las barreras del tiempo. La Brecha Digital cuantifica la diferencia existente entre países, sectores y personas que tienen acceso a los instrumentos y herramientas de la información y la capacidad de utilizarlos y aquellos que no lo tienen.

- Participación Ciudadana: Sin participación no hay interacción y sin interacción no hay innovación. $Y$ sin participación y capacidad para la innovación no habrá un verdadero desarrollo para una estrategia de gobierno electrónico.

DESARROLLO

Análisis de gobierno electrónico a nivel internacional

Se realizó un análisis de las experiencias de Gobierno Electrónico en Países Desarrollados como Reino Unido cuya visión es Modernizar el Gobierno, mediante la incorporación de tecnologías informáticas con el objeto de mejorar la calidad de vida de las personas y la productividad de las empresas. En Australia se estableció la Oficina Nacional para la Economía de la Información, (National Office for the Information Economy). Esta unidad tiene como responsabilidad desarrollar, coordinar y monitorear políticas macro, incluyendo la aplicación de nuevas tecnologías para proveer servicios de Gobierno Electrónico. Es también responsabilidad de esta unidad la de crear conciencia sobre los beneficios y asuntos relacionados con Gobierno Electrónico. También se creó la Oficina para el Gobierno en Línea, (Office for Government 
Online - OGO). OGO tiene un rol de coordinador y facilitador para asistir a las agencias del Gobierno Federal $y$ departamentos en la implantación de servicios de Gobierno Electrónico y en la actualidad, se está implementando un programa para mejorar los servicios policiales Ilamado "Delta" que permite desde la localización automática de vehículos hasta el manejo de casos criminales con herramientas informáticas. La base es una red $60 \%$ terrestre y $40 \%$ satelital.

Por otro lado, en Canadá se tiene como visión convertir al gobierno canadiense como usuario modelo de la tecnología de la información y las comunicaciones, actuando como catalizador del desarrollo y la innovación en el uso de la autopista de la información en toda la sociedad y economía. La organización responsable de la gerencia en el proceso de cambio se denomina Chief Information Officer at TBS (CIOB). Adicionalmente, dentro de $\mathrm{CIOB}$, se creó una oficina especial de control de proyectos para la Agenda de Gobierno en Línea. Esta oficina tiene como responsabilidad principal proveer métricas y esquemas de control de proyectos comunes a los diferentes departamentos, así como monitorear el progreso de los mismos. Cada departamento en el Gobierno de Canadá es responsable de hacer la reingeniería necesaria de sus procesos para proveer servicios en línea.

En Francia se designó un comité interministerial, dirigido por el Primer Ministro cuya función es hacer progresar la Agenda de Gobierno Electrónico. Cada ministerio es responsable de implementar las medidas que acuerden el Comité y el Ministerio de Economía, Finanzas e Industria, www.finances.gov.fr, tiene un rol clave en el financiamiento de nuevos proyectos. Cada ministerio es responsable de identificar sus proyectos dirigidos a Gobierno Electrónico.

Para Singapur el desarrollo de servicios electrónicos es prioridad en este país, dada la intención del gobierno de convertirse en un centro de comercio electrónico. Las estructuras de gobierno de Singapur tienen algunas diferencias marcadas con respecto a otros países en este benchmarking dado que Singapur es un estado-ciudad, con una población de menos de cuatro millones de habitantes y el número de funcionarios que prestan servicios públicos es relativamente pequeño (60.000 personas). La visión de Singapur es ser líder en Gobierno Electrónico para servir mejor al país y a los ciudadanos en la sociedad del conocimiento planteando una serie de estrategias para alcanzar sus metas. Todo el desarrollo de Gobierno Electrónico es liderado por una sola oficina, Government Chief Information Office (GCIO), con un staff de 400 personas que trabajan sobre iniciativas globales o centrales.

En el mismo orden de ideas el Ministerio de Educación de Suecia implementó un programa de tres años, llamado itis, con el que se da entrenamiento a más de $60 \mathrm{mil}$ maestros en el uso de las nuevas tecnologías. Este proceso va acompañado por la dotación de equipos de computación y aperturas de cuentas de correo electrónico a cada uno de los participantes del programa itis. Otorgaron conexión a la red de Internet a las escuelas y ofrecen el servicio a todas las municipalidades del país.

En Israel el Parlamento Israelí (Knesset) está implementando un proceso de modernización y reingeniería, llamado eparlamento, que le permitirá brindar respuestas en tiempo real sobre legislación, supervisión del gobierno y representación de la opinión ciudadana. $Y$ en Alemania la ciudad-estado de Bremen, resultó ganadora 
de una competencia multimedia orientada a desarrollar conceptos para la seguridad y la legalidad de los servicios online para ciudadanos, basados en firmas digitales. El ciudadano podrá firmar con su llave privada, transmitiendo al gobierno los datos mediante un sistema de encriptación.

Por otro lado, Baja Sajonia es el primer gobierno estatal alemán en usar firmas digitales con tarjetas inteligentes para el proceso de autenticación de su personal interno, trazándose como objetivo la reducción de costos por transacciones internas e incrementar el trabajo "sin papeles".

En cuanto al Análisis del Gobierno-e en América Latina, se pudo identificar el aumento de las iniciativas de gobierno-e en América Latina que a su vez ha traído como consecuencia el desarrollo de una corriente de investigaciones en el área. Existen diferentes esfuerzos académicos y de investigación promocionados por organismos multilaterales como la Organización de los Estados Americanos (OEA), Programa de las Naciones Unidas para el Desarrollo y el Centro Latinoamericano (CEPAL), entre otros. Otros son de corte académico/investigativo como las Facultad Latinoamericana de Ciencias Sociales (FLACSO). Todos ellos brindan apoyo a los países y a otras organizaciones para el estudio y desarrollo del gobierno electrónico. En esta sección se consideran algunos trabajos recopilados de diferentes fuentes, relacionadas con organismos multilaterales $u$ organizaciones académicas/investigativas, con el fin de aportar distintas visiones del gobierno-e en Latinoamérica.

El gobierno electrónico como tema y como práctica ha ido ocupando un importante espacio en el ámbito de la modernización de los estados latinoamericanos, ya sea en la gestión pública o en la relación Estado-ciudadanía. Como concepto, comenzó a ser utilizado desde la segunda mitad de los noventa para dar cuenta de las transformaciones producidas por la incorporación de las TIC en el quehacer de las instituciones públicas. La instalación de la temática del gobierno-e no es casual ni espontánea, cuenta con importantes antecedentes y es parte de una tendencia internacional. El debate sobre el gobierno-e está vinculado a discusión global y a las reflexiones sobre el rol del Estado. A partir de los años ochenta comienza a ser conocida una nueva tendencia en la administración pública. En algunos países se comienzan a incorporar conceptos y nociones desde el mundo del comercio electrónico (ecommerce). La nueva gestión pública establece una nueva relación entre lo político a lo gerencial, propone la descentralización, la reducción de costos y la flexibilidad laboral introduce la noción de competencia interna y externa, la externalización de servicios y, quizás la más importante de todas, postula un cambio de énfasis desde los procesos a los resultados, lo que implica incorporar medición de desempeño y orientación a los ciudadanos (clientes del Estado, desde esta perspectiva).

Actualmente el tema del gobierno-e se ha instalado en los debates y en las agendas políticas de América Latina con mucha fuerza, propiciando encuentros entre los sectores públicos y privados para llegar a acuerdos de intercambio tecnológico, creando leyes y políticas públicas relativas a su implantación, incentivando su utilización por los ciudadanos e incorporando su estudio en todos los niveles educativos. El gobierno-e es entendido como una de las formas de expresión de la sociedad de la información, así como un ámbito en el proceso de modernización del Estado. 
Establece el uso estratégico e intensivo de las tecnologías de la información y la comunicación, tanto en las relaciones del propio sector público entre sí, como en las relaciones de los órganos del Estado con los ciudadanos, usuarios y empresas del sector privado.

\section{RESULTADOS Y DISCUSIÓN}

\section{Modelos teóricos para la medición del desarrollo de gobierno electrónico}

Los gobiernos han trabajado para formular modelos de desarrollo que integren estrategia, personal, procesos y tecnología al igual que planes de acción efectivos para trazar una implementación exitosa.

El modelo basado en fases ha ganado popularidad entre los gobiernos y consiste en la descripción de fases de desarrollo de eGobierno y el análisis estructural de las transformaciones. Generalmente, las fases iniciales de la evolución de la e-gobierno implican la publicación o la catalogación de información en la Web. Subsecuentemente, los sistemas internos se conectan a interfaces en línea y así los ciudadanos pueden conectarse electrónicamente con el gobierno. El objetivo principal en el desarrollo de los portales gubernamentales es brindar un punto de acceso único donde los ciudadanos puedan configurarlos de acuerdo a características seleccionadas por ellos mismos. Con esto, un gobierno puede establecer relaciones de uno a uno con los ciudadanos así como canales de comunicación bidireccionales. En la etapa final de la evolución, desaparecerá la percepción de departamentos gubernamentales distintos y el público verá al gobierno como una sola entidad.

A continuación, se describen de manera breve las fases del modelo de desarrollo de e-gobierno de la ONU publicado en el reporte titulado "Benchmarking eGovernment": A Global Perspectiva. 2001. Assessing the Progress of the UN Member States".

- Emerging Presence: Un determinado país decide incursionar en el mundo del e-gobierno. Existe una presencia Web formal, pero limitada, a través de algunos pocos sitios Web gubernamentales, los cuales proporcionan a los ciudadanos información organizacional o política. Los sitios pueden incluir información de contacto, como por ejemplo números telefónicos o direcciones de servidores públicos.

- Enhanced Presence: La presencia en línea de un país empieza a expandirse con el aumento de sitios oficiales Web. Contiene información más dinámica y especializada que es actualizada frecuentemente; los sitios contienen ligas hacia otras páginas oficiales. Están disponibles en línea publicaciones gubernamentales, legislación y boletines de noticias; así como servicios de búsqueda en línea y direcciones de correo electrónico. Y finalmente puede existir un sitio en línea del gobierno nacional que contenga ligas hacia otros ministerios 0 departamentos.

- Interactive Presence: La presencia en la Internet de un país se expande enormemente con acceso a un amplio número de instituciones y servicios gubernamentales. Existen niveles más sofisticados de interacciones formales entre los ciudadanos y los servidores públicos, como por ejemplo el correo electrónico o áreas de comentarios por Internet. Se encuentran disponibles en línea las aplicaciones de formas al igual que la posibilidad de enviarlas por 
Internet, así como la capacidad de búsqueda de bases de datos especializadas.

- Transactional Presence: Son posibles las transacciones completas y seguras como por ejemplo la obtención de visas, pasaportes, actas de nacimiento $y$ defunción, licencias, permisos, pago en línea de servicios como multas, honorarios de registro de automóviles e impuestos. Pueden existir las firmas digitales, para facilitar a los negocios y su interacción con el gobierno. Se hacen presentes los sitios seguros y claves de acceso a usuarios.

- Seamless or fully integrated: Se cuenta con la capacidad de acceso instantáneo a cualquier servicio dentro de un único portal. Las líneas de demarcación ministerial/departamental/agencia ya no existen en el ciber-espacio. Los servicios son clasificados de acuerdo a las necesidades comunes.

\section{Indicadores internacionales relacionados con gobierno electrónico}

En el marco del Observatorio para la Sociedad de la Información en Latinoamérica y el Caribe (OSILAC), proyecto desarrollado por la División de Desarrollo Productivo y Empresarial de la CEPAL y en colaboración con el Grupo de Trabajo sobre medición de las Tecnologías de la Información y Comunicación (TIC) de la Conferencia Estadística de las Américas (CEA) de la CEPAL, desarrollaron una propuesta para la medición de indicadores de gobierno electrónico en la región, tomando como base el trabajo realizado por la Comisión Económica de África. La propuesta regional consta de diez indicadores básicos o clave, y siete indicadores extendidos. Esta propuesta fue discutida por representantes de las
Oficinas Nacionales de Estadística (ONE) en el marco del Quinto Taller sobre la Medición de la Sociedad de la Información en América Latina y el Caribe, realizado en abril de 2009 en Río de Janeiro (Naser, 2009). En la discusión se resaltó la importancia de dar impulso a la medición del Gobierno Electrónico en nuestros países, pues este constituye un tema clave en el funcionamiento de los Estados, que ha mostrado importantes resultados en el aumento en la transparencia y la eficiencia de los gobiernos en los países donde se ha venido implementando. Se sugiere además que la recolección de información comience por el Gobierno Central, y con el tiempo extender el levantamiento de datos a niveles de gobierno regional y local.

A continuación, se muestran los diez indicadores claves y los siete extendidos propuestos por el Grupo TIC de la CEA y la OSILAC, (Sunkel, 2009) cabe hacer notar que aún no existen datos conocidos de estos indicadores, sin embargo, es importante dar a conocer los indicadores, ya que en algún esfuerzo futuro se podría trabajar en obtener esta información.

\section{Indicadores clave}

- CEG1 Porcentaje de empleados en organizaciones gubernamentales que usan computadoras rutinariamente para su trabajo.

- CEG2 Porcentaje de empleados en organizaciones gubernamentales que usan internet rutinariamente para su trabajo.

- CEG3 Porcentaje de empleados en organizaciones gubernamentales que usan correo electrónico rutinariamente para su trabajo. 
- CEG4 Porcentaje de organizaciones gubernamentales con presencia en internet en su propio sitio web o en el sitio web de otra entidad.

- CEG5 Porcentaje de organizaciones gubernamentales con redes corporativas (lan, wan, intranet y extranet).

- CEG6 Porcentaje de organizaciones gubernamentales con estándares de interoperabilidad.

- CEG7 Porcentaje de organizaciones gubernamentales con acceso a internet, según tipo de acceso (banda angosta, banda ancha fija y banda ancha móvil).

- CEG8 Porcentaje de organizaciones gubernamentales que ofrecen plataformas de servicios a usuarios, según tipo de plataforma disponible: web, teléfono fijo, fax y teléfono móvil.

- CEG9 Porcentaje de organizaciones gubernamentales que ofrecen servicios en línea, según tipo de actividad.

- CEG10 Porcentaje de organizaciones gubernamentales que ofrecen servicios en línea, según tipo de servicio.

\section{Ranking de gobierno electrónico a nivel internacional}

El índice de desarrollo del gobierno electrónico de las Naciones Unidas (2012), (EGDI, por sus siglas en inglés) es un indicador compuesto que mide la predisposición y la capacidad de las administraciones nacionales para utilizar las tecnologías de la información y de las comunicaciones en la prestación de los servicios públicos. Se basa en un estudio integral de la presencia en línea de los 193 Estados Miembros que evalúa las características técnicas de los sitios web nacionales, así como las políticas de gobierno electrónico y las estrategias que en general aplican los sectores específicos en la prestación de los servicios esenciales.

La evaluación llevada a cabo por la ONU DEAS es una medición relativa, es decir que califica el desempeño del gobierno electrónico de los países respecto del de los demás países; no es una medición absoluta. Los resultados están tabulados y combinados con un conjunto de indicadores que comprenden la capacidad de un país para participar en la sociedad de la información, sin la cual los esfuerzos de desarrollo del gobierno electrónico tienen una utilidad inmediata limitada.

Matemáticamente, el EGDI es el promedio ponderado de tres puntuaciones normalizadas de las dimensiones más importantes del gobierno electrónico, a saber: alcance y calidad de los servicios en línea, el estado de desarrollo de la infraestructura en telecomunicaciones y el capital humano inherente (Chahuara, 2015)). Cada uno de estos conjuntos de índices constituye en sí una unidad medida compuesta que puede extraerse y analizarse de forma independiente. EGDI = (Índice de servicios en línea) + (Índice de infraestructura en telecomunicaciones) + (Índice de capital humano).

\section{Índice de servicios en línea}

Con el fin de identificar diferencias en los aspectos determinantes del desarrollo del gobierno electrónico entre países grandes y pequeños, y teniendo en cuenta los desafíos adicionales a los que se enfrentan los países grandes, se llevó a cabo la evaluación de los efectos de la población y de la superficie territorial en los países con una población de al menos 100 millones de habitantes y se tomaron en consideración aspectos resaltantes tales como: Servicios de 
información emergente: las páginas web gubernamentales proporcionan información sobre política pública, gobernanza, legislación, reglamentación, documentación pertinente y tipos de los servicios gubernamentales proporcionados. Servicios de información mejorada: los sitios web gubernamentales facilitan la comunicación unidireccional mejorada o la comunicación bidireccional simple entre el gobierno y el ciudadano, tales como la descarga de formularios de los servicios y aplicaciones gubernamentales. Servicios transaccionales: los sitios web gubernamentales participan con los ciudadanos en una comunicación bidireccional, incluidas la solicitud y recepción de sugerencias sobre las políticas, los programas, la reglamentación gubernamental, etc. Los sitios web gubernamentales procesan transacciones que no son de carácter financiero; por ejemplo, votación electrónica, descarga y carga de formularios, presentación de declaraciones de impuestos en línea o solicitud de certificados, licencias y permisos.

Servicios integrados: los sitios web gubernamentales han cambiado la forma en que los gobiernos se comunican con los ciudadanos. Ellos solicitan de manera activa información y opiniones de los ciudadanos que utilizan la Web 2.0 y otras herramientas interactivas. Los servicios electrónicos y las soluciones electrónicas traspasan los departamentos y ministerios de manera uniforme. La información, los datos y el conocimiento se transfieren de los organismos gubernamentales mediante aplicaciones integradas.

\section{Índice de infraestructura en telecomunicaciones}

El índice de infraestructura en telecomunicaciones es un promedio aritmético compuesto de cinco indicadores: número estimado de usuarios de Internet por cada 100 habitantes; número de línea telefónica fija y principal por cada 100 habitantes; número de suscriptores de telefonía móvil por cada 100 habitantes; y número de instalaciones de banda ancha fija por cada 100 habitantes. En cada caso, la fuente de información principal es la Unión Internacional de Telecomunicaciones (UIT).

\section{Índice de capital humano}

El índice de capital humano es un promedio ponderado compuesto de dos indicadores: la tasa de alfabetización de adultos y el coeficiente bruto de inscripción en la instrucción primaria, secundaria y terciaria combinado; la tasa de alfabetización de adultos compone dos tercios el índice y el coeficiente bruto de inscripción combinado compone un tercio. La fuente principal de los datos de estos indicadores es la Organización de las Naciones Unidas para la Educación, la Ciencia y la Cultura.

\section{Ámbito Nacional}

\section{Contexto de gobierno electrónico en el estado venezolano}

El Gobierno Electrónico en Venezuela significa el aprovechamiento de las tecnologías de Información y Comunicación para aumentar la inclusión de amplios sectores que han estado al margen de la acción social del Estado. Las Tecnologías de Información no sólo pueden propiciar la transformación del Estado, maximizando la eficiencia de la administración pública, también son un mecanismo para aumentar la transparencia y garantizar la seguridad de la nación. Arturo Gallegos. Director General de la Oficina de Tecnologías de Información del Ministerio de Ciencia y Tecnología. 2005. 
Mendoza (2005) manifiesta que:

El Gobierno Electrónico no es la tecnificación de la burocracia existente, sino que tiene que ver con la inserción de la creatividad y la innovación en la Administración Pública, para crear procedimientos asociados a viejos y nuevos servicios, que resuelvan $y$ simplifiquen la vida del ciudadano.(s/p).

Presidente del CNTI, 2006 (Berrizbeitia, 2005). "El Gobierno Electrónico es un medio para que el Estado dé respuestas oportunas a las nuevas exigencias de los ciudadanos, reduzca la burocracia, la duplicación de esfuerzos y los retrasos innecesarios"

Líder del Proyecto Gobierno en Línea del CNTI, 2005 (Riveros y Mendoza, (2005). En estas percepciones se destaca el aspecto social asociado al gobierno electrónico y a las Tecnologías de Información y Comunicación, que no son vistas sólo como medios para ampliar el alcance de la gestión gubernamental y política. En teoría, en la visión oficial del gobierno electrónico en Venezuela se busca ir más allá de la mejora en la gestión del Estado, su transformación y el ofrecimiento de servicios oportunos y efectivos; se busca incorporar iniciativas orientadas a aumentar la transparencia del Estado, garantizar la seguridad de la nación, fomentar el desarrollo local, la contraloría social, la participación en la gestión pública, la soberanía tecnológica, la democracia participativa y la inclusión social, partiendo de la necesidad inicial de formar a los ciudadanos y funcionarios en el significado y uso de las tecnologías asociadas a las soluciones de gobierno electrónico.

\section{Beneficios del gobierno electrónico}

Para los ciudadanos el desarrollo de la tecnología y la rápida caída en el precio de las comunicaciones y los equipos de computación han transformado la vida de muchas personas. Se han establecido nuevos servicios, y los servicios existentes son provistos de maneras novedosas. Aprovechados al máximo, estos servicios proporcionan el beneficio de: 1. Mejor acceso, con servicios disponibles donde y cuando existe una demanda. 2. Despacho a través de diversos medios: en taquilla, a través de un centro de atención telefónica y en-línea. 3. Segmentación del mercado, con servicios ajustados para cubrir las necesidades de grupos dentro de un mercado particular. 4. Mejor respuesta al feedback sobre el contenido y la calidad de los servicios. 5. Agrupamiento de servicios en torno a episodios de vida o eventos comunes.6. Análisis comprehensivo de datos referentes a patrones de uso. Y 7 . Participación de los usuarios en el rediseño y mejora de servicios.

Por parte de las empresas las nuevas tecnologías han transformado la manera de operar en los negocios. Una de las formas claves en que las empresas han aplicado las técnicas de comercio electrónico es en el manejo de su relación con clientes y proveedores (Customer Relationship Management and Supply Chain). Los organismos del sector público deben hacer lo mismo si quieren obtener los mismos beneficios en términos de reducción de costos y mejores logros.

El sector público no solo interactúa con las empresas como un cliente 0 un proveedor. Adicionalmente, es el responsable de un amplio rango de funciones reguladoras $y$ de soporte que pueden ser facilitadas. 
Y por último para el Sector Público los nuevos métodos de trabajo también ofrecen beneficios potenciales para el funcionamiento interno del gobierno. Estos incluyen ganancia en eficiencia y efectividad por un mejor uso y manejo de la información, ya sea en el soporte al establecimiento de políticas o en la administración de programas. Las tecnologías de Intranet ofrecen la posibilidad de establecer base de conocimientos $y$ mecanismos de trabajo inter-departamental. Las conexiones de Extranet entre organizaciones pueden permitir llevar a cabo gestiones de forma rápida y económica.

\section{Modelo adoptado en la República Bolivariana de Venezuela para la medición de gobierno electrónico.}

El desarrollo del gobierno electrónico debe asumirse como un proceso evolutivo que comprende al menos cuatro fases: presencia, interacción, transacción y transformación. Estas fases no son interdependientes ni tampoco necesitan que termine una para que comience la otra. Cada una de ellas tiene distinto objetivo y requiere distintas exigencias en términos de costos, necesidades de conocimiento y nivel de uso de las TIC.

Otros modelos incluyen una quinta fase que unos denominan "Integración entre administraciones", otros "Gobierno electrónico holístico" y algunos "Administración relacional" o "Democracia electrónica" (Consorcio del proyecto Atlántico, 2003) o "Participación Democrática" (Cardona, 2002).

En la descripción de cada una de estas fases se debe tomar en cuenta lo siguiente:

- Presencia: En esta primera etapa el gobierno tiene presencia en Internet a través de la divulgación de sus sitios web o portales. En esta instancia los organismos ponen a disposición información básica de manera rápida y directa. No existe mayor relación con ciudadanos y empresas debido a que la información puesta en línea no posibilita la interacción.

- Interacción: En la etapa de interacción es posible una comunicación más directa entre los ciudadanos y los organismos. Estos no solo brindan información, sino que están preparados para recibir opiniones y establecer una comunicación con la población a través del correo electrónico, envío de formularios, de comentarios de opinión o de foros.

- Transacción: Con la transacción, una vez completada la etapa anterior, los organismos brindan a los ciudadanos la posibilidad de iniciar, realizar el seguimiento y finalizar cualquier trámite en línea, sin tener que ir personalmente a la dependencia correspondiente. Los sitios web de los organismos están preparados para ofrecer sus trámites y servicios como un complemento de la atención "cara a cara" en las oficinas. Hasta esta fase, el avance se genera fundamentalmente por un salto tecnológico. Es decir, no se generan implicaciones en las estructuras organizacionales o funcionales de las agencias de la administración pública. (Cardona, 2002).

- Transformación: En la cuarta fase, que corresponde a la transformación, el salto es cultural, lo cual genera un reto mayor para su implementación porque implica una redefinición de los servicios y de la operación de la administración pública, creando una integración total entre agencias $y$ entre niveles regionales, así como con el sector privado, las 
creando una integración total entre agencias y entre niveles regionales, así como con el sector privado, las organizaciones no gubernamentales y el ciudadano, permitiendo servicios cada vez más personalizados (Cardona, 2002).

- Participación democrática: Representa el nivel más sofisticado de las iniciativas en línea del gobierno, puede ser caracterizada por una integración de las interacciones con empresas, ciudadanos y otras administraciones. El gobierno estimula la toma de decisiones participadas y está dispuesto a implicar a la sociedad en la red en un diálogo de doble dirección. A través de características interactivas tales como blogs, foros y otros, el gobierno solicita activamente opiniones y participación a los ciudadanos y los integra en el proceso interno de toma de decisiones (Tricas, 2007).

Sin embargo, para poder medir la participación democrática se debe tener la certeza de que las cuatro etapas anteriores posean un alto grado de madurez en materia de Gobernanza Electrónica, es por ello que la presente investigación se considera cuatro fases de la madurez de Gobierno Electrónico propuesta por Michel Backus (2001).

\section{Fases del goblerno electrónice}

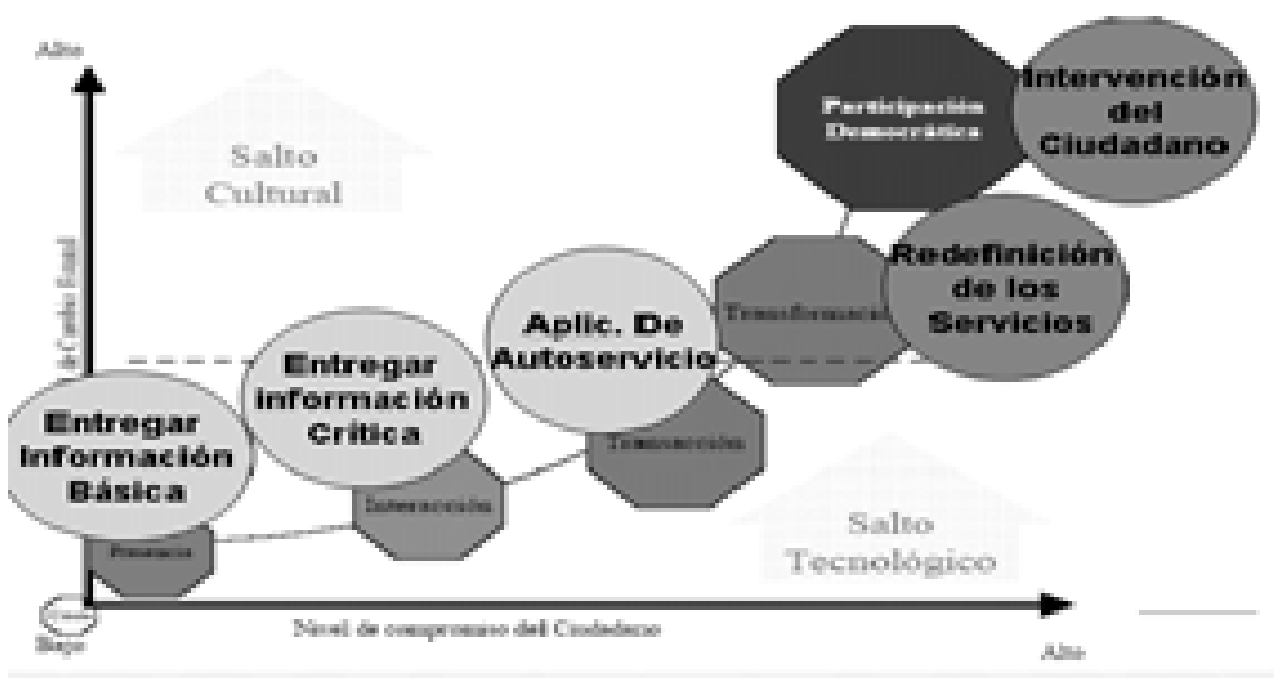

Gráfico 1. Las Cinco Etapas de Gobierno Electrónico

En el marco de las relaciones se presenta la siguiente caracterización de cada una de ellas:

1. Gobierno - Ciudadana o Ciudadano (GC): es la entrega de productos, servicios, información o transacciones a las ciudadanas o ciudadanos.
2. Gobierno - Empresa (G-E): es la entrega de productos, servicios, información o transacciones a empresas o negocios con personalidad jurídica.

3. Gobierno - Gobierno (G-G): son iniciativas destinadas a satisfacer los requerimientos de información, 
servicios, transacciones o productos entre las instituciones del Estado.

4. Gobierno - Trabajadora o Trabajador (G-T): es la entrega de información, productos, transacciones o servicios de desarrollo profesional y atención al potencial humano de las instituciones del gobierno.

Para el logro de estas relaciones, la implementación de gobierno electrónico pasa por un proceso evolutivo, donde se distinguen cuatro dimensiones de desarrollo: Información, interacción, transacción y transformación. Las cuales no son dependientes una de la otra, tampoco se considera necesario que termine una para iniciar la siguiente dimensión.

\section{Gobierno electrónico en Venezuela: puesta en práctica y logros}

En Venezuela se observa que a través del uso de internet se observa un serio esfuerzo por la presentación de Portales, que integren y faciliten el acceso a los diferentes entes gubernamentales a nivel nacional $y$ local. Internet ha servido, también, para difundir y propagar la información de los Programas Sociales Gubernamentales (misiones) y en particular de los programas de gobierno que apuntan a la inclusión de todos los sectores en el uso de las tecnologías (alfabetización tecnológica).

Como un logro a destacar, orientado a la implantación de la democracia electrónica, se puede resaltar la automatización del sistema de votación en el país, así como el interés del Gobierno Nacional en introducir la temática del gobierno electrónico en los acuerdos regionales, a nivel de América Latina, en una nueva concepción de la integración regional que incorpora los aspectos sociales, científico-tecnológicos, ambientales y políticos además de los económicos. Para ello Venezuela se adhiere a la iniciativa de desarrollo de la Red de Líderes de Gobierno Electrónico de América Latina y El Caribe (Red GEALC), promocionado por la División de Innovaciones y Alianzas para el Desarrollo de la AICD/OEA y el Instituto de Conectividad de las Américas (ICA), como un espacio de intercambio de conocimiento, expertos y soluciones en todos los temas relacionados con el gobierno electrónico, con el fin de facilitar la colaboración y el esfuerzo compartido entre los gobiernos de la región.

Los esfuerzos en materia de gobierno-e en Venezuela se pueden evidenciar en la existencia de portales que proveen información institucional y en algunos casos permiten realizar transacciones:

- Servicios de Gobierno Electrónico: a) Solicitud de Divisas - CADIVI (http://www.cadivi.gov.ve). b) Declaración y pago de impuestos SENIAT (http://www.seniat.gov.ve/). c) Portal Gobierno - Gobierno en Línea (http://www.gobiernoenlinea.ve/). d) Seguro Social - $\quad$ IVSS (http://www.ivss.gov.ve/). e) Gobierno Electrónico Local - Alcaldías Digitales (http://www.alcaldias.gov.ve). f) Identificación Ciudadana - SAIME (http://www.saime.gov.ve/), entre otros.

- Servicios de Democracia Electrónica: Implantación del sistema de votación automatizado (www.cne.gob.ve).

- Planes y Proyectos Sociales: a) Misión Ciencia

(http://www.misionciencia.gob.ve). b) Plan Nacional de Alfabetización Tecnológica. c) Infocentros (http://www.infocentro.gov.ve/). Centros Bolivarianos de Informática y Telemática (CBIT). e) Otras Misiones ( http://www.misionvenezuela.gov.ve/) 
CONCLUSIONES

Dentro de los elementos comunes en los diferentes enfoques utilizados por los países desarrollados, se identifican como mejores prácticas las siguientes:

La conformación de una unidad central, entre cuyas responsabilidades destacan: a) Asumir el liderazgo, b) Definir estrategias de tecnologías de información, c) Asesorar a los demás ministerios/departamentos, d) Comunicar y crear "cultura" de Gobierno Electrónico en los ciudadanos, e) Identificar iniciativas globales, es decir, aquellas iniciativas que "crucen horizontalmente" las diferentes estructuras del gobierno. f) La designación de responsables en cada ministerio/departamento, entre cuyas responsabilidades destacan: Identificar oportunidades, realizar los ajustes de procesos necesarios, implementar las iniciativas locales. g) Una unidad especial encargada de: Coordinar las diferentes iniciativas locales, así como a sus respectivos responsables, Esta responsabilidad debe tener como principales objetivos: evitar duplicidad de esfuerzos y crear sinergia, h) Implementar iniciativas globales. Como iniciativas globales más importantes destacan: Implementar proyectos de infraestructura en tecnologías de información, desarrollar habilidades relacionadas con Gobierno Electrónico en los empleados de gobierno, monitorear el progreso tanto global como local y proveer métricas y esquemas de trabajo comunes a todos los entes de gobierno. i) Los elementos que más resaltan en la implementación del Gobierno Electrónico en el mundo son: El objetivo más importante es mejorar la prestación de servicios a la comunidad, la reducción de costos se convierte en un objetivo secundario o menos importante, la implementación del Gobierno Electrónico se hace más efectiva desde jurisdicciones de organización política unitaria (Juntas Parroquiales, Municipios, Estados), hacia organizaciones más complejas (Estados como Unidad), existe un organismo central que promueve y coordina las distintas actividades asociadas al Gobierno Electrónico, el organismo central establece estrategias de mediano y largo plazo para el desarrollo de proyectos particulares, el primer paso o proyecto a ejecutar, es el mejoramiento de las infraestructuras de telecomunicaciones, las naciones destinan fondos para la implementación de las nuevas tecnologías y comienza un proceso serio de depuración de base de datos y consolidación de las mismas.

Gobierno Electrónico en los países latinoamericanos no escapan de esa realidad y debido a eso sus gobiernos están trabajando en conjunto con organismos multilaterales para implantar la tecnología y el conocimiento necesarios para llevarlo a cabo. Aunque el gobierno-e debe ser un tema central de discusión multidisciplinario y transdiciplinario en las universidades e institutos de investigación se nota un desfase entre el acelerado crecimiento del gobiernoe y el avance en materia educativa al respecto: casi todas las investigaciones analizadas son de fuentes independientes o de organismos gubernamentales 0 multilaterales.

Además, salvo pocos casos como el de Brasil, no se evidencia una tendencia al gobierno-e para las organizaciones intergubernamentales lo cual dificulta la integración regional. También existe una marcada separación entre los países de la región que están en proceso de transición de un Estado centralista burocrático a un Estado participativo inclusivo utilizando las buenas prácticas de gobierno-e, en particular se 
advierte que existen países como Chile, México y Brasil y Argentina que han superado la etapa informacional y ya presentan soluciones en el ámbito transaccional; otros, como Colombia, Uruguay y Perú se encuentran en una fase fundacional creando reglamentaciones, diseñando proyectos y poniendo en práctica experiencias tanto informacionales como transaccionales que permitan evaluar el impacto que tiene en el ciudadano estas iniciativas. Seguramente, al igual que ha ocurrido en otros países esta fase será superada en la medida que se decanten soluciones prácticas y útiles de las inoficiosas. Otro grupo, como los países centroamericanos se encuentran aún en fase de evaluación del proceso de transferencia hacia el gobierno-e. Ninguno de los países de Latinoamérica hasta ahora, ha superado la fase transaccional a la participativa, salvo contadas experiencias del voto electrónico en Brasil y Venezuela; sin embargo, no se han explorado otras formas de participación activa y consuetudinaria de la ciudadanía como la generación de políticas públicas por la vía de la colaboración en línea, entre otras.

Los proyectos más exitosos en gobiernoe también han coincidido en que han tenido un fuerte liderazgo gubernamental al más alto nivel, en algunos casos los propios presidentes o primeros ministros como principales promotores. Así mismo la experiencia demuestra que ha sido fundamental que los compromisos institucionales adquiridos sean de largo plazo, es decir, que no dependan de cambios de ministros o funcionarios intermedios. Del mismo modo los planes de operación también deben ser sustentables a largo plazo. Otra lección que nos dejan los países con mayores logros en esta materia se refiere al liderazgo dentro del gobierno. Hay coincidencia en que debe haber una instancia que lidere las transformaciones, ya sea un ministerio o un consejo o comité de alto nivel.

Dado que el gobierno-e afecta a toda la administración pública y es un ámbito esencialmente transversal, se requiere de una institucionalidad que le de respaldo, poder y visibilidad a las iniciativas en esta materia. Cuando depende de comités de coordinación de múltiples servicios públicos, tienden a debilitarse los esfuerzos. Las comisiones asesoras han demostrado ser una buena práctica para el desarrollo de planes estratégicos o para el seguimiento de la implementación de iniciativas de gobierno-e. Estas comisiones generalmente están compuestas por altos funcionarios de gobiernos, ejecutivos del sector privado, universidades y organizaciones de la sociedad civil. (Causa y otros, 2005).

De acuerdo a los espacios internacionales en materia de indicadores de TIC, se puede decir que Venezuela no ha tenido una participación activa y en de acuerdo al ranking de Gobierno Electrónico 2012 nos posicionamos en el lugar número 71 con un índice servicios en línea de 0.585 , un índice de infraestructura en telecomunicaciones de 0.3215 y el índice de capital humano 0,8705 , estando por debajo de Colombia, Chile, Uruguay, México, Argentina, Brasil y Panamá y por encima de Dominica, el Salvador, Paraguay, Bolivia, Honduras y Nicaragua.

Parte del análisis realizado sobre Venezuela en cuanto a Gobierno Electrónico es la existencia de leyes regulatorias para la implementación rigurosa de GE, también se presenta la inexistencia de una estrategia que regule $y$ direccione de una manera formal las acciones que deben realizarse con respecto a las TIC y los beneficios al 
ciudadano común en esta materia con miras a lograr una gestión pública transparente y confiable, sin embargo pese a dicha situación, se han suscitado cambios positivos en diversas instituciones gubernamentales que han logrado la satisfacción y reconocimiento por parte de los habitantes del país de una mejor gestión.

\section{REFERENCIAS}

Backus, Michael (2001). E-governance in Developing Countries del Instituto Internacional para la. Comunicación y el Desarrollo de Holanda, IICD, Research Report N. 3, April 2001. Documento en línea. Disponible en http: www.ftpiicd.org/files/research/reports /report3.pdf Consulta: 2006, Octubre 25

Berrizbeitia, J. L. (2005). La Sociedad del Conocimiento en Venezuela. Anuario ININCO, (17)

Cardona, D. (2002). El gobierno electrónico: Una revisión desde la perspectiva de la prestación de servicios. En: I Congreso Catalán de Gestión Pública. Barcelona, España

Causa, A., Finquelievich, S., Finquelievich, D., Goldstein, R., Ahón, E. I., Jacob, P., y Prince, A. (2005). e-Política y e-Gobierno en América Latina. Buenos Aires.

Chahuara, P. (2015). Propuesta para la Construcción de Índices Comparativos en los Países Miembros de la CITEL (No. 32)

Centro Nacional de Tecnologías de Información (2010). Gobierno electrónico en la administración pública. Caracas, Venezuela.

Cimoli, M., y CEPAL, N. (2005). Heterogeneidad estructural, asimetrías tecnológicas y crecimiento en América Latina
Consorcio del proyecto Atlántico (2003), "La Administración Pública", España

Mendoza, B. (2005). Un Portal para el Gobierno Electrónico. Directorio de Gobierno Electrónico Venezuela Ministerio de Ciencia y Tecnología (MCT). Disponible en: http://www.gobiernoenlinea.gob.ve/dir ectorioestado/experiencias.html

Naciones Unidas (2007). Modelo multidimensional de medición del gobierno electrónico para América Latina y el Caribe. Santiago de Chile, Chile.

Naciones Unidas (2012). Estudio de las Naciones Unidas sobre el gobierno electrónico, 2012. Gobierno electrónico para el pueblo. Nueva York, Estados Unidos.

Naser, A. (2009). Gobierno Electrónico: Indicadores. Instituto Latinoamericano y del Caribe de Planificación Económica y Social (ILPES). Recuperado de: http://www. cepal. org/ilpes/noticias/paginas/0/40660/alej andra_naser_INDIC ADORES.pdf.

Riveros, V. S., y Mendoza, M. I. (2005). Bases teóricas para el uso de las TIC en Educación. Encuentro educacional, 12(3).

Sandoval Almazán, Rodrigo y Gil -García, J. Ramón (2009). Propuesta de evaluación para portales de gobierno electrónico basada en el enfoque teórico evolutivo. México, México

Sunkel, G. (2009). Avances y desafíos en el desarrollo y uso de indicadores TIC en educación

Tricas-Lamana, F. (2007). El gobierno electrónico: servicios públicos y participación ciudadana, Documento de trabajo 109/2007. Fundación Alternativas 